\title{
Conservação de água em shopping centers: análise das percepções dos atores envolvidos nos projetos e gestão, com o uso do processo de hierarquia analítica
}

\author{
Water conservation in shopping centers: analysis of \\ the perceptions of the actors involved in projects and \\ management, using the analytical hierarchy process
}

João Luiz Calmon' ${ }^{\oplus}$, Celso Silva Bastos* ${ }^{1 *}$

\section{RESUMO}

As áreas urbanas buscam novas opções de serviço de água, para garantir o abastecimento. A literatura acadêmica versa sobre o pensamento de que a crise hídrica está relacionada ao crescimento urbano; no entanto, o fator predominante é o aumento do consumo, independentemente do crescimento populacional, pois fatores comportamentais devem ser revistos a partir de simples hábitos diários. Os empresários não focam apenas nas questões de economia ou da discussão ambiental, de mais vale a garantia da autonomia, pois a insegurança hídrica é cada vez mais crescente. Empreendimentos como os shopping centers poderão consumir volumes de água equivalentes ao consumo de 5 mil pessoas. Para saber as percepções dos atores envolvidos no design e na gestão de shopping centers, este trabalho utilizou o método AHP, com a aplicação de questionários e resultados combinados. Dados resultantes demonstram que o conhecimento dos envolvidos nem sempre corresponde aos conceitos de conservação de água e que uma melhor sinergia entre a ciência e o mercado deve estar mais próxima.

Palavras-chave: conservação de água; shoppings centers; construções sustentáveis; fontes alternativas de água; método Processo de Hierarquia Analítica; sustentabilidade; reúso.

\begin{abstract}
Urban areas seek new water service options, to ensure the supply. The academic literature deals with the thought that the water crisis is related to urban growth; however, the predominant factor is the increased consumption, regardless of population growth, as behavioral factors should be reviewed based on simple daily habits. Entrepreneurs do not focus only on economic issues or on top of environmental discussion, as well ensuring autonomy, as water insecurity is increasingly growing. Enterprises such as shopping malls may consume water volumes equivalent to the consumption of 5,000 people. In order to know the perceptions of the actors involved in the design and management of shopping malls, this work used the AHP method, with the application of questionnaires and combined results. Resulting data demonstrate that the knowledge of those involved does not always correspond to the concepts of water conservation and a better synergy between science and the market should be more forthcoming.
\end{abstract}

Keywords: water conservation; shopping malls; sustainable buildings; alternative sources of water; Analytical Hierarchy Process method; sustainability; reuse.

\section{INTRODUÇÃO}

O mundo enfrenta uma grave crise hídrica. Em regiões onde a disponibilidade de água doce é limitada, a reciclagem e a reutilização têm ocorrido de forma crescente, compensando o uso e reduzindo a descarga de águas residuais. Nunes (2006) afirma que há carência de pesquisas nos segmentos comerciais, de serviços e entretenimentos, corroborada pela opção em desenvolver uma pesquisa para um empreendimento comercial de grande porte, como um shopping center, neste caso, visto que a maioria dos trabalhos existentes voltados para conservação de água está focada em indústrias, instituições e hospitais e edifícios corporativos, o que foi constatado pelos autores desta pesquisa e, assim, retificou a escolha do tema.

'Universidade Federal do Espírito Santo Ringgold Standard Institution - Vitoria (ES), Brasil.

*Autor correspondente: celsobastos10@gmail.com

Conflitos de interesse: os autores declaram não haver conflito de interesses.

Financiamento: nenhum.

Recebido: 08/05/2019 - Aceito: 2O/06/2O2O - Reg. ABES: 20190149 
Com base nas pesquisas realizadas pelos autores no portal periódico da Capes, foram localizados 7 artigos de autores de referência, sendo 4 tratando de shoppings e 3 sobre edificações comerciais. O Quadro 1 apresenta os autores referentes aos artigos citados. Já com relação a artigos analisando o uso do AHP em conservação da água em shopping centers não foi localizado nenhum, o que corrobora a pesquisa, havendo uma lacuna a ser preenchida em relação a empreendimentos comerciais de grande porte, sendo esses grandes consumidores de água.

Os shoppings centers formam uma categoria que consome grande quantidade de água em todo o Brasil, devido ao crescente número de usuários e ao consumo unitário. Prédios comerciais são potenciais consumidores de água e de 50 a $90 \%$ do uso é destinado para descargas sanitárias e torre de arrefecimento. Fontes alternativas devem ser exploradas, para redução do consumo de água potável (BOYLE, 2005; FREIRE, 2011).

No caso desta pesquisa, o método AHP é projetado para estruturar um processo de decisão em um cenário afetado por múltiplos fatores. Trata-se de uma metodologia aceita pela comunidade científica internacional, devido a sua flexibilidade na tomada de decisões, a qual envolve múltiplos critérios na solução de problemas complexos (BOTTERO; COMINO; RIGGIO, 2011).

Destaca-se que, em termos gerais, o método AHP é uma hierarquia de variáveis e tem o propósito de gerenciamento tanto dos valores tangíveis, como intangíveis, segundo diferentes critérios e atores envolvidos em uma problemática ambiental. Considera-se que uma das principais vantagens do AHP é a possibilidade de incorporar aspectos qualitativos na análise dos dados. No caso deste trabalho, a percepção dos especialistas e profissionais envolvidos é determinante para avaliar e, até mesmo, quantificar o cenário da conservação da água em grandes empreendimentos comerciais, como os shoppings centers.
Neste caso, o método AHP foi considerado o mais indicado, devido a sua capacidade em incorporar a subjetividade envolvendo múltiplos atores com interesses conflitantes. Dessa forma, considerou-se a possibilidade de uma ferramenta que desse ao autor deste trabalho uma visão holística sobre o que pensam e como atuam os envolvidos relacionados de modo geral ao planejamento de um empreendimento de grande porte, como o Shopping Vila Velha. Por último, usou-se o software Expert Choice, que, por meio de modelos matemáticos e comparações por pares, serviu de base para a elaboração de questionários, montados por meio de uma estrutura em níveis (objetivo, critérios, subcritérios e alternativas), formulando gráficos individuais por participantes e combinados entre todos. O propósito foi quantificar sobre o que discorriam os agentes envolvidos e como o seu pensamento está relacionado ao dos especialistas responsáveis pela elaboração das normas e que dá sustentação científica à questão.

Considera-se que a tomada de decisão deve buscar uma opção entre várias, a qual apresente melhor desempenho, avaliação e acordo entre as expectativas de quem decide, que, por sua vez, deve escolher a melhor alternativa disponível do curso de ação que a pessoa deve seguir.

\section{METODOLOGIA}

Para elaboração da pesquisa e tomada de opiniões dos participantes, foi aplicado o método Analytic Hieracty Process (AHP), sendo este o mais popular do Multiple Criteria Decision Making (MCDM) para formular e analisar decisões. O AHP foi desenvolvido por Thomas L. Saaty, a partir de 1977, como um método de análise de decisões, por meio da estruturação dos componentes da decisão, ou seja, esse método deriva escala de razões para comparações vinculadas, além de possibilitar a análise de diversas alternativas,

Quadro 1 - Artigos publicados.

\begin{tabular}{|c|c|c|c|c|c|c|}
\hline Autores & Título & Ano & Fonte & $\begin{array}{l}\text { Shopping ou edifício } \\
\text { comercial }\end{array}$ & País & Afiliação \\
\hline Bint et al. & $\begin{array}{l}\text { "Alternative water sources in New } \\
\text { Zealand's commercial buildings" }\end{array}$ & 2019 & Water Supply & Comercial & Nova Zelandia & $\begin{array}{l}\text { BRANZ / Institute of } \\
\text { Environmental Science } \\
\text { and Research }\end{array}$ \\
\hline $\begin{array}{l}\text { Cook, Sharma } \\
\text { e Gurung }\end{array}$ & $\begin{array}{l}\text { "Evaluation of alternative water sources } \\
\text { for commercial buildings: A case study } \\
\text { in Brisbane, Australia" }\end{array}$ & 2014 & $\begin{array}{l}\text { Resources, Conservation } \\
\text { and Recycling }\end{array}$ & Comercial & Australia & $\begin{array}{l}\text { CSIRO Land and Water } \\
\text { Austrália, Highett / } \\
\text { Griffith University }\end{array}$ \\
\hline $\begin{array}{l}\text { De Gois, Rios } \\
\text { e Costanzi }\end{array}$ & $\begin{array}{l}\text { "Evaluation of water conservation and } \\
\text { reuse: A case study of a shopping mall in } \\
\text { southern Brazil" }\end{array}$ & 2015 & $\begin{array}{l}\text { Journal of Cleaner } \\
\text { Production }\end{array}$ & Shopping & Brasil & $\begin{array}{l}\text { Universidade } \\
\text { Tecnológica Federal do } \\
\text { Paraná }\end{array}$ \\
\hline Joustra e Yeh & $\begin{array}{l}\text { "Framework for net-zero and net-positive } \\
\text { building water cycle management" }\end{array}$ & 2015 & $\begin{array}{l}\text { Building Research and } \\
\text { Information }\end{array}$ & Comercial & EUA & $\begin{array}{l}\text { University of South } \\
\text { Florida }\end{array}$ \\
\hline $\begin{array}{l}\text { Wang, Chang } \\
\text { e Nunn }\end{array}$ & $\begin{array}{l}\text { "Lifecycle assessment for sustainable } \\
\text { design options of a commercial building } \\
\text { in Shanghai" }\end{array}$ & 2010 & $\begin{array}{l}\text { Building and } \\
\text { Environment }\end{array}$ & Comercial & China & $\begin{array}{l}\text { Shandong University, } \\
\text { China / Faithful Gould, } \\
\text { United Kingdom }\end{array}$ \\
\hline $\begin{array}{l}\text { Sousa, Silva e } \\
\text { Meireles }\end{array}$ & $\begin{array}{l}\text { "Performance of water efficiency } \\
\text { measures in commercial buildings" }\end{array}$ & 2019 & $\begin{array}{l}\text { Resources, Conservation } \\
\text { and Recycling }\end{array}$ & Shopping & Portugal & $\begin{array}{l}\text { University of Lisbon / } \\
\text { University of Aveiro }\end{array}$ \\
\hline $\begin{array}{l}\text { Sousa, Silva e } \\
\text { Meireles }\end{array}$ & $\begin{array}{l}\text { "Technical-financial evaluation of } \\
\text { rainwater harvesting systems in } \\
\text { commercial buildings-case ase studies } \\
\text { from Sonae Sierra in Portugal and Brazil" }\end{array}$ & 2018 & $\begin{array}{l}\text { Environmental Science } \\
\text { and Pollution Research }\end{array}$ & Shopping & Portugal & $\begin{array}{l}\text { University of Lisbon / } \\
\text { University of Aveiro }\end{array}$ \\
\hline
\end{tabular}

Fonte: primária 
e as compara velozmente (TRENTIM, 2012). O método AHP foi projetado para auxiliar em um processo em que há cenários com múltiplos decisores. Utilizando-o é possível desmembrar um problema complexo em subpartes descritas, segundo um grau de hierarquia (BOTTERO; COMINO; RIGGIO, 2011; JAGTAP; BEWOOR, 2017). Para Ribeiro e Costa (1999, p. 7), o método baseia-se em três níveis de pensamento analítico (Figura 1).

A hierarquia deve seguir a seguinte ordem: propósito geral do problema, objetivo, os critérios e, por fim, as alternativas. A prioridade é decompor os fatores de acordo com suas inter-relações e relações subordinadas, e os fatores são reunidos em diferentes níveis. O problema então é organizado na forma de um modelo estrutural analítico de vários níveis e poderá ser resolvido destinando pesos relativos aos diferentes níveis, do mais baixo ao mais alto (que representa o objetivo geral), ou à ordem dos méritos (KURTTILA et al., 2000).

Para a definição das prioridades, os tomadores de decisão dão início ao processo de atribuir importância relativa aos elementos em cada nível. Cada critério e subcritério tem pesos diferentes, para cada nível da hierarquia. Da mesma maneira, as alternativas variam de forma diversa em cada critério (CHEN, 2006). Os passos a seguir para determinar as prioridades são:

- Julgamentos paritários: aqui é proposta a comparação entre pares por meio de uma matriz de comparação pareada. Os resultados são representados na matriz de julgamento, que é usada para fazer as comparações entre os pares e representa a importância dos elementos entre os níveis atual e anterior (WANG et al., 2018). Os elementos da matriz são calculados pela Equação 1:

$a i j>0, a i j=\frac{1}{a i f}, a i j=1$

Em que:

A = a matriz de comparação;

$\mathrm{n}=$ as alternativas;

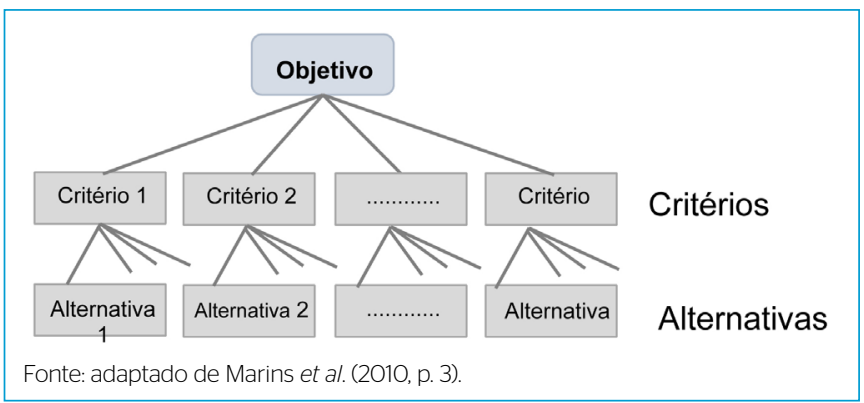

Figura 1 - Estrutura hierárquica básica. aij = a medida de preferência da alternativa na linha i quando se compara com a alternativa da coluna $j$.

Tanto i quanto j variam de 1 a n (Equação 2).

$\mathrm{A}\left[\begin{array}{ccc}1 & a 12 \cdots & a 1 n \\ \vdots & \vdots & \vdots \\ 1 / a n 1 & 1 / a n 2 \cdots & 1\end{array}\right]\left|\begin{array}{ccc}1 / a 21 & 1 & \cdots a 2 n \\ \vdots & & \end{array}\right|$

Os valores dos elementos na matriz de julgamento seguem as determinações de Saaty e variam de 1 a 9, como mencionado na Tabela 1, a seguir:

- Normalização das matrizes: segundo Costa, Rodrigues e Felipe (2008, p. 8), para normalizar é necessário "somar cada coluna da matriz, dividida por todos os elementos de cada coluna pelo somatório referente à coluna";

- Cálculo das prioridades médias locais (PMLs): são as médias aritméticas das colunas e linhas dos quadros;

- Cálculo das prioridades globais: o intuito dessa etapa é identificar um vetor de prioridade global (PG) que seja capaz de armazenar a prioridade interligada a cada alternativa em relação ao foco principal. Para isso, é necessário combinar os PMLs no vetor de prioridades global. Consistência lógica: para ter utilidade, o método AHP tem de ter consistência de julgamento par a par. A consistência é definida pela fórmula: aij $\mathrm{x}$ ajk = ajk. Quando a matriz de comparação é consistente, o maior valor próprio é ( $\lambda \max )$ será igual a n. Quando há consistência, ocorre um desvio $(\lambda \max -n)$ e, por sua vez, essa medida é dividida por n-1, resultando na média dos autovetores (ABEDI; TORABI; NOROUZI, 2013). O consistency índex (CI) é obtido por meio da Equação 3:

$\mathrm{CI}=\left(\lambda_{\max }-n\right) /(n-1)$

Para saber se as avaliações são consistentes, é necessário calcular o consistency ratio (CR). Esse índice é a razão entre IC e o random index (RI). A fórmula então ficaria da seguinte forma: $\mathrm{CR}=\mathrm{CI} / \mathrm{RI}$. O índice aleatório é o grau de consistência que surge de maneira automática quando se completa a matriz com os valores na escala de 1-9 (Tabela 2) (MACHARIS et al., 2004).

O limite do CR aceito é menor que 0,10 , caso contrário será necessário refazer o processo de avaliação para encontrar a consistência (TEMIZ; CALIS, 2017). Por meio do método AHP é que se apresentam os dados empíricos desta pesquisa.

\section{APLICAÇÃO DO MÉTODO ANALYTIC HIERACTY PROCESS}

Para que fossem conhecidas as percepções dos atores envolvidos no processo de construção e operação de um shopping center, foram elaborados questionários baseados com o método AHP. Os questionários foram respondidos por

Tabela 1 - Escala numérica de Saaty.

\begin{tabular}{|c|c|c|c|c|c|c|c|c|}
\hline \multicolumn{9}{|c|}{ Escala de comparação dos Critérios com as Alternativas A e B } \\
\hline 9 & 7 & 5 & 3 & 1 & 3 & 5 & 7 & 9 \\
\hline Extremamente & Bastante & Muito & Pouco & $\begin{array}{c}\text { Igual } \\
A=B\end{array}$ & Pouco & Muito & Bastante & Extremamente \\
\hline
\end{tabular}

Fonte: primária. 
Tabela 2 - Índice aleatório.

\begin{tabular}{|c|c|c|c|c|c|c|c|c|c|c|c|c|c|c|c|}
\hline $\mathrm{n}$ & 1 & 2 & 3 & 4 & 5 & 6 & 7 & 8 & 9 & 10 & 11 & 12 & 13 & 14 & 15 \\
\hline RI & 0 & 0 & 0,58 & 0,90 & 1,12 & 1,24 & 1,32 & 1,41 & 1,45 & 1,49 & 1,52 & 1,54 & 1,56 & 1,58 & 1,59 \\
\hline
\end{tabular}

Fonte: Macharis et al. (2004).

quatro gestoras e 12 empresas, sendo que as respostas de uma gestora correspondem ao procedimento adotado em vários shoppings que estão sob sua gestão, chegando uma delas a operar 40 shoppings no país. Os questionários dessa categoria de profissionais foram respondidos por 40 participantes, sendo 20 arquitetos e 20 engenheiros, todos profissionais atuantes no estado, com no mínimo 5 anos no exercício na profissão. Os questionários foram divididos em duas etapas para cada categoria de participantes. O primeiro questionário faz a comparação dos critérios com subcritérios, sendo o segundo questionário a comparação de critérios com as alternativas. As categorias foram definidas entre profissionais (arquitetos e engenheiros) e gestoras e empresas, que são os segmentos ligados ao saneamento, ao abastecimento, às gestões sustentáveis e às operadoras de shoppings. Eles foram encaminhados aos participantes selecionados e, tão logo devolvidos respondidos, tiveram os resultados inseridos nos quadros de valores gerados no software Expert Choice, programa específico para aplicação dos resultados matemáticos e geração de gráficos de resultados parciais e coletivos.

Conforme a metodologia do AHP, foi criada uma estrutura em níveis compostos por objetivos, critérios, subcritérios e alternativas, base para desenvolvimento dos questionários, como apresentado na Figura 2.

No primeiro questionário, denominado questionário 1 , foram realizadas comparações entre os critérios e, em seguida, comparação entre critérios e subcritérios, gerando gráficos individuais por participantes ou gráficos combinados que representam o resumo de todos os julgamentos dos participantes.

No segundo questionário, denominado questionário 2 , as comparações se repetem entre os critérios, pois é preciso manter os 3 níveis da estrutura, conforme o método AHP, e, em seguida, são feitas as comparações entre critérios e alternativas, gerando, da mesma forma, gráficos por participantes e gráficos combinados.

O objetivo foi analisar, em síntese, os resultados combinados de todos os atores, para posteriormente comparar as respostas com o pensamento dos especialistas e poder contribuir de forma a direcionar todos os conhecimentos a um senso comum, em prol da eficiência e do desempenho da gestão da água em shopping centers.

A Figura 3 apresenta a resposta do julgamento entre os critérios, envolvendo todos os participantes (gestoras, empresas e profissionais [arquitetos e engenheiros]), sendo aplicado o questionário 1 .

Fica claro, como mostrado na Figura 3, que, na comparação entre os critérios, o ambiental foi o de maior peso no julgamento de todos os participantes combinados, ficando com $30,9 \%$, seguido pelo econômico $(22,3 \%)$ e, depois, pelo técnico (16,5\%), pelo operacional $(9,8 \%)$ e, por fim, pelo social $(15 \%$.)

Lembrando que os subcritérios que formam o critério ambiental são a redução da pressão nos mananciais, a redução de produtos químicos, a redução de efluentes líquidos, o reúso e a reciclagem e, por fim, a energia incorporada.
Nota-se grande preocupação, por parte de todos os participantes, com as questões ambientais, mas será que no desenvolvimento do empreendimento existem ações projetuais incorporadas que efetivamente levem a esse resultado ambiental positivo, ou é um desejo esperado, mas sem o conhecimento e a ação necessários capaz de levar a esse resultado?

No questionário 2, aplicado a todas as categorias (Figura 4), define-se o resultado entre os critérios e as alternativas, considerando-se que no caso as alternativas são os subcritérios que formam os critérios.

Em uma análise parcial dentro do critério técnico, o subcritério distribuição foi considerado como o item de maior importância, com $5,2 \%$, seguido por captar $(4,5 \%)$, armazenar $(4,3 \%)$ e tratar (2,5\%). Já para o critério operacional o subcritério regulagem periódica fica à frente como o de maior relevância $(4,9 \%)$, vindo a seguir controle de perdas e desperdícios (4,1\%), dificuldade de operação $(2,9 \%)$, dificuldade de manutenção $(3,9 \%)$ e, por fim, capacitação profissional (1,3\%), nessa ordem decrescente.

No critério econômico, o subcritério considerado de maior importância foi menor custo na conta de água e esgoto (7,5\%), seguido por custo de operação $(4,4 \%)$, custo de manutenção $(3,9 \%)$, investimento $(2,4 \%)$, custo de energia $(2,1 \%)$, valorização imobiliária $(1,2 \%)$ e, por último, imagem perante o público $(0,7 \%)$.

Dentre os itens do critério ambiental, a redução da pressão nos mananciais foi o subcritério de maior impacto entre os profissionais (14\%), seguida por reúso e reciclagem $(9,1 \%)$, redução de efluentes líquidos $(3,7 \%)$, energia $(2,2 \%)$ e, por fim, redução de produtos químicos com $1,9 \%$, nessa ordem decrescente.

Por último, e não menos importante, vem o julgamento do critério social, ficando incentivo ao desenvolvimento local como o subcritério de maior peso $(6,6 \%)$, seguido por benefícios à comunidade $(4,6 \%)$ e, por último, geração de emprego (3,8\%). Somando-se o resultado de todos os subcritérios de cada critério, chega-se a $100 \%$ dos valores julgados e o de maior valor de julgamento, pela ótica de todos os participantes combinados, foi "redução da pressão nos mananciais", representando $14 \%$, seguido por "reúso e reciclagem", com $9,1 \%$. Um resultado que surpreende, pois espera-se dos empresários que os resultados econômicos sejam os de maior relevância, considerando-se a construção do empreendimento e os resultados financeiros em operação; no entanto, a pesquisa apontou para ações ambientais como de maior valor de julgamento.

A Figura 5 apresenta a resposta do julgamento entre os critérios, envolvendo todos os participantes (gestoras, empresas e profissionais [arquitetos e engenheiros]), sendo aplicado o questionário 2 (critério $\times$ critério e critérios $\times$ alternativas [subcritérios]).

Para o julgamento das fontes alternativas, a Figura 6 apresenta como item de maior importância, na visão dos participantes combinados, a água cinza.

Para uma análise parcial entre cada critério e as alternativas, os resultados estão apresentados nas Figuras 7, 8, 9, 10 e 11. 


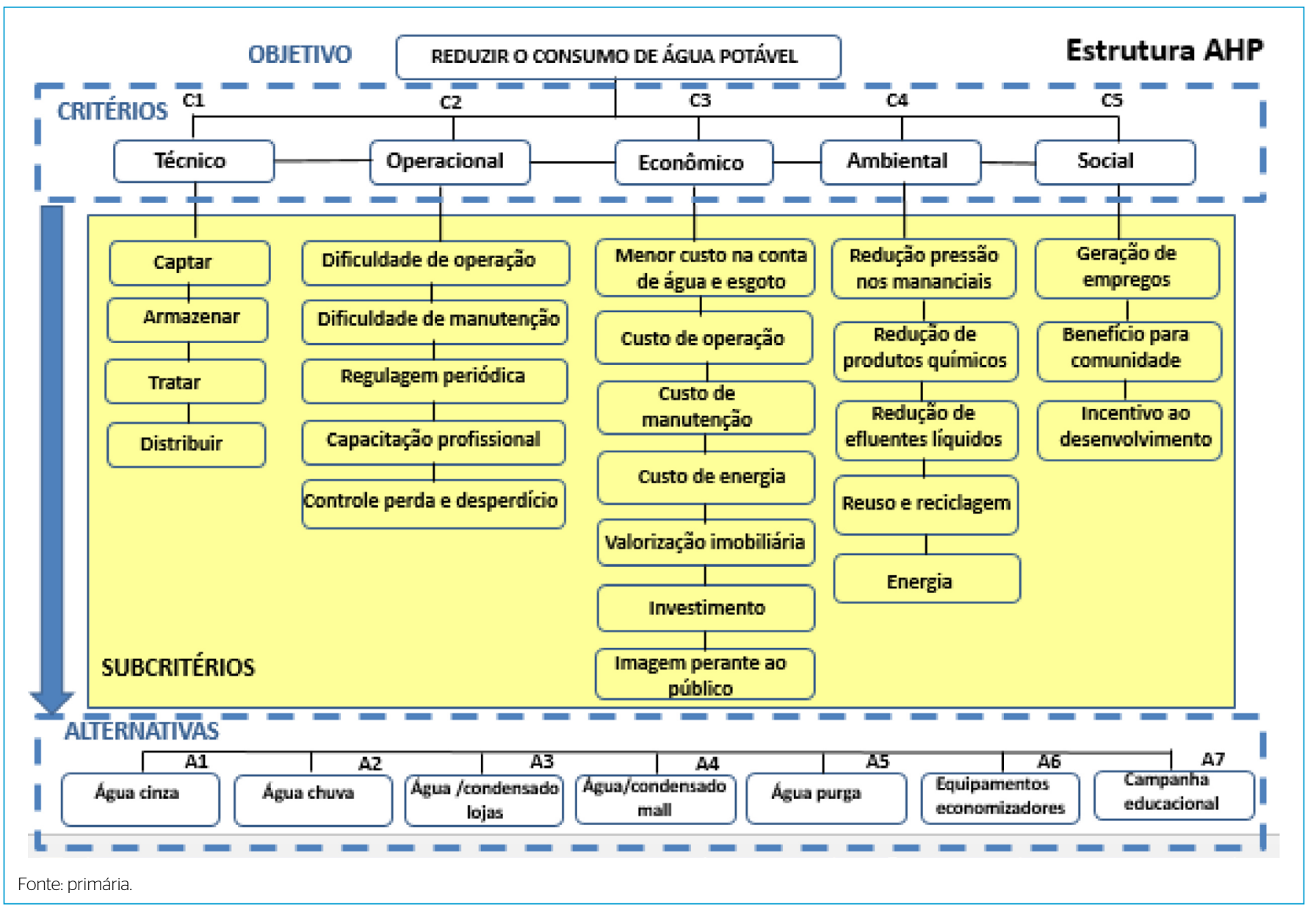

Figura 2 - Estrutura do Método AHP.

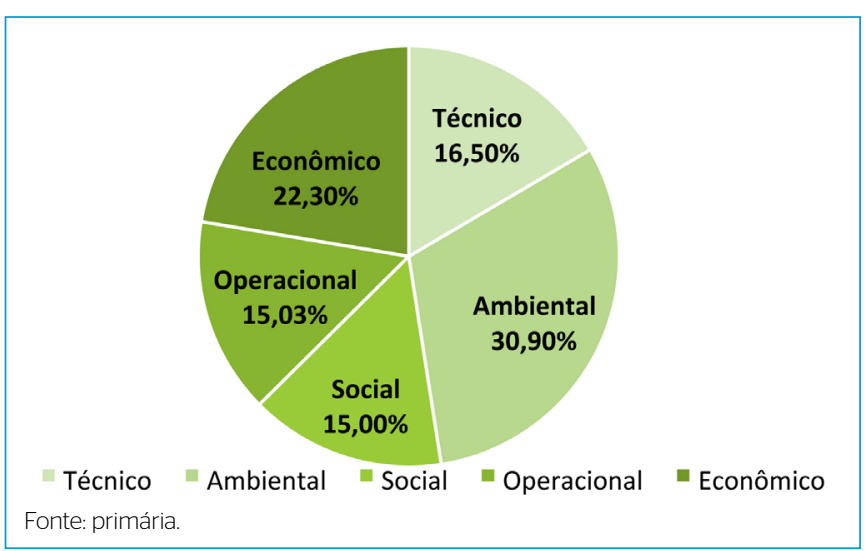

Figura 3 - Critério × critério/profissionais, gestoras, empresas.

\section{RESULTADOS}

A percepção dos envolvidos (profissionais, gestoras e empresas) foi resultante do pensamento de todos combinados, sendo que a aplicação dos questionários foi para saber como pensam e agem os participantes, por meio da experiência de cada um, no sentido de expressar o raciocínio nas fases de concepção e criação, quando envolvidos no desenvolvimento de projeto ou gestão de um shopping.
A Figura 12 expressa o resultado resumido de cada subcritério relativo ao critério de referência.

O subcritério "redução da pressão sob os mananciais" (critério ambiental) foi o item de maior peso, com $14 \%$, pela ótica dos profissionais, gestoras e empresas, ficando o subcritério "menor custo na conta de água e esgoto" com 7,50\% (critério econômico), seguido do subcritério "incentivo ao desenvolvimento local", com 6,60\% (critério social), depois pelo subcritério "distribuir", com 5,20\% (critério técnico), e, por último, pelo subcritério "regulagem periódica" $(4,9 \%)$, no critério operacional.

A concepção de um shopping se inicia no projeto arquitetônico, que parte de um programa de necessidades levantadas a partir do estudo técnico e econômico da viabilidade do negócio e dos resultados pretendidos. É notório que difundir a ciência, ao alcance dos atores, seja de extrema valia para um resultado superior. Conteúdos de pesquisas e conversas com o empresariado devem ser levados à frente para serem aplicados.

\section{DISCUSSÃO E CONCLUSÕES}

$\mathrm{O}$ ato de incorporar medidas de gestão da oferta e demanda, com o uso de fontes alternativas e maior capacidade de operação e manutenção, compõe um dos elementos que poderão garantir um abastecimento estratégico e independência. 


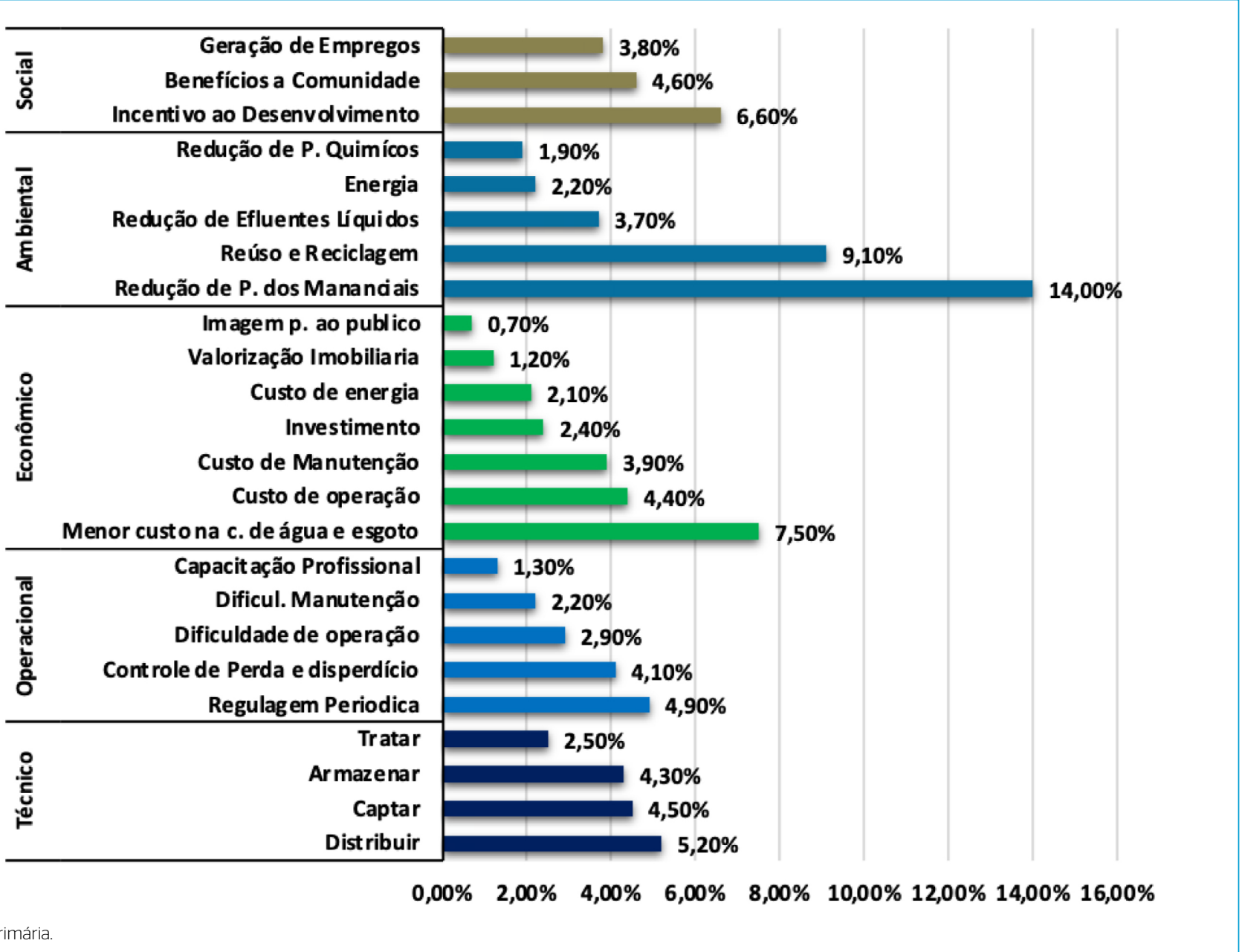

Fonte: primária

Figura 4 - Critério × Alternativas (subcritérios)/profissionais, gestoras, empresas.

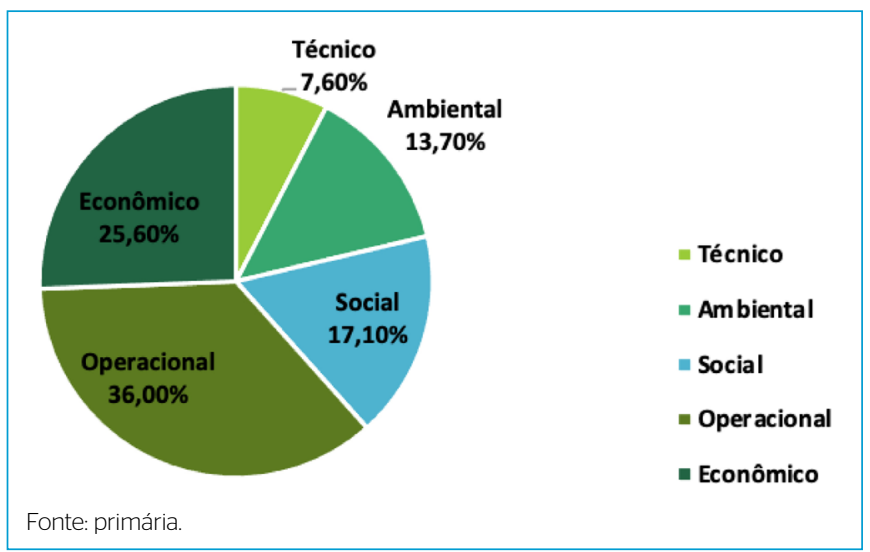

Figura 5 - Critério × critério/profissionais, gestoras, empresas.

Dentro do pensamento de especialistas, o que poderá, de fato, proporcionar a redução do consumo de água potável em um shopping ou qualquer empreendimento é o monitoramento e o controle do consumo, reunindo aspectos tecnológicos e comportamentais na gestão. Essa ação deverá preceder investimentos em equipamentos e o uso de fontes alternativas.
Só com essa atividade já se poderá ter ganhos na redução da água, pois não adianta inserir novas tecnologias sem antes controlar o que já existe (KIPERSTOK, 2008).

Uma brigada de bombeiros hidráulicos deverá estar apta para ações rápidas e contínuas de manutenção e regulagem dos equipamentos economizadores, calibrando-os em relação à vazão ótima para cada finalidade. Para isso, um bom projeto em parcimônia com as questões de conservação de água irá contribuir muito para as ações e reações da equipe responsável pela boa gestão do shopping.

Os dados de consumo e as metas de redução devem ser acompanhados diariamente pelos administradores e expostos ao público, como fonte de incentivo ao usuário consciente, o que poderá promover a sensibilização do usuário, trazendo ganhos econômicos, sociais e ambientais, por meio da construção permanente educacional.

As práticas de reúso e aproveitamento de água de chuva ou condensação são consideradas processos de substituição de fontes, com utilidades diferentes dentro se um shopping, afirma Zanella (apud Bello, 2013, p. 59), e merecem um estudo criterioso, devido aos custos de instalação, operação e manutenção.

Para Bint et al. (2019), a captação de água de chuva ou a reutilização de água cinza deve ser baseada em um sistema tarifário apropriado, o que levaria 


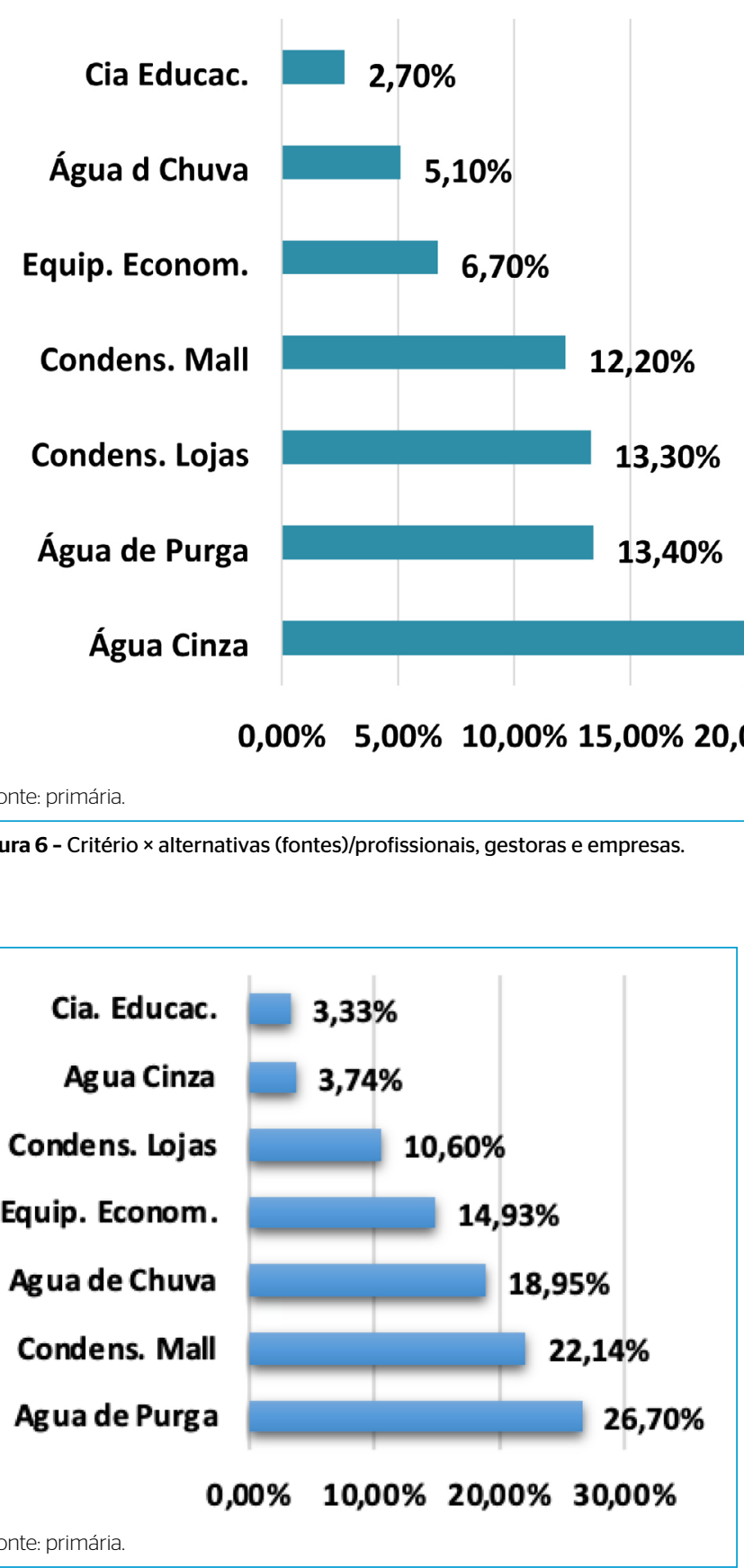

Figura 7 - Critério técnico.

a uma diminuição dos custos. Os autores ainda descrevem que é importante o conhecimento e treinamento de gerentes de construção, para melhor operar um sistema de gestão de água sustentável.

Já para Adulis (apud Bello, 2013, p. 60), além da redução no consumo, é preciso considerar melhores formas de reúso dentro de um shopping, sendo que o ideal seria um reaproveitamento de $100 \%$, fechando o ciclo hídrico, mas a sustentabilidade está relacionada a tornar-se viável. Ressalta ainda que os produtos e as lojas que levam em conta a sustentabilidade têm tido reconhecimento e preferência pelos consumidores e investidores ao procurarem um shopping

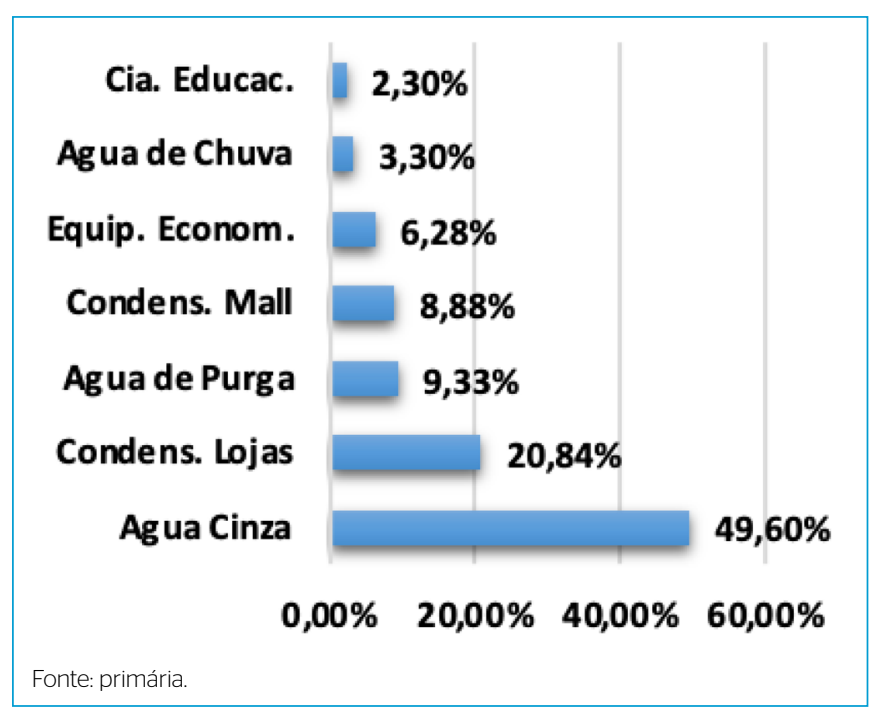

Figura 8 - Critério operacional.

para abertura de uma filial, o que traz um diferencial aos empreendimentos que adotam esses conceitos. Decerto o resultado favorável refletirá nas contas de águas e energia e nas taxas de condomínios.

Em seu estudo, Sousa, Silva e Meireles (2019) descrevem que o custo de instalações sanitárias apresenta maior economia anual de água, porém tem maior período de retorno (15 anos), pois precisa de alto investimento e manutenção. Já para a captação de águas pluviais o retorno é de aproximadamente 10 anos. O sistema de reutilização de águas cinzas tem retorno em mais de dois anos. 


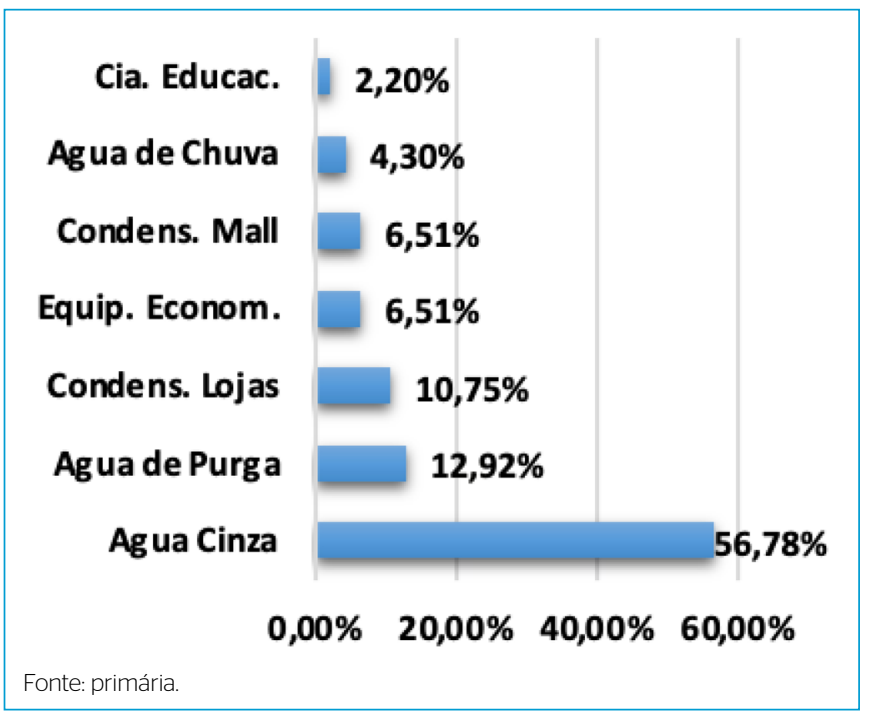

Figura 9 - Critério econômico.

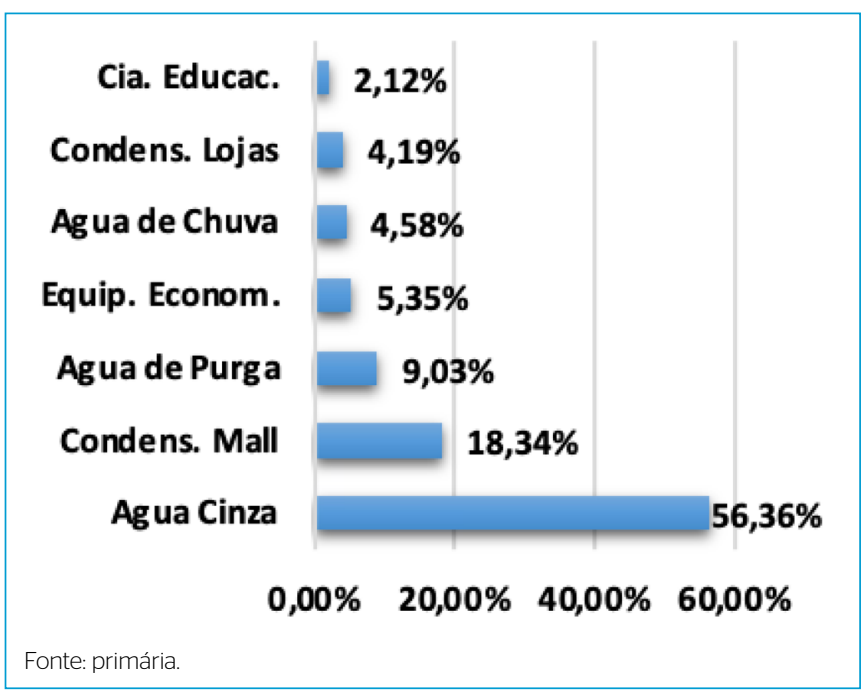

Figura 10 - Critério ambiental.

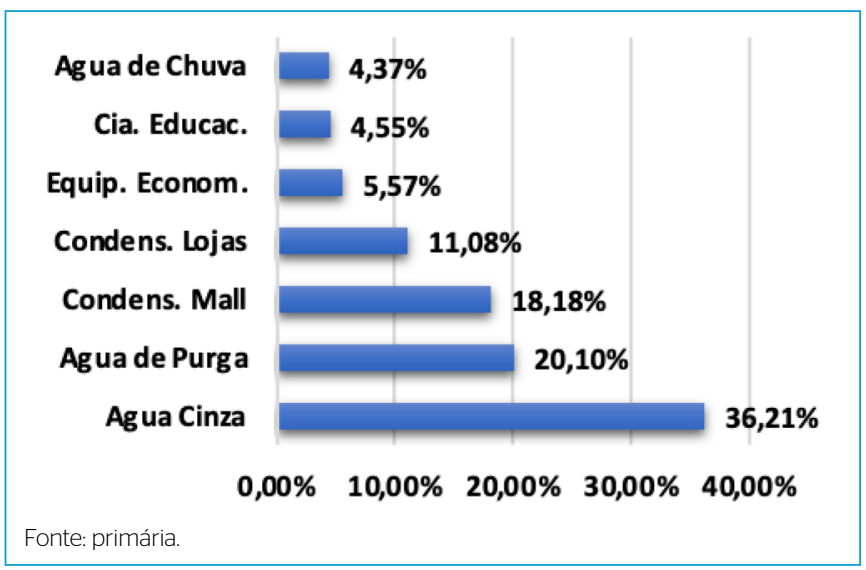

Figura 11 - Critério social.

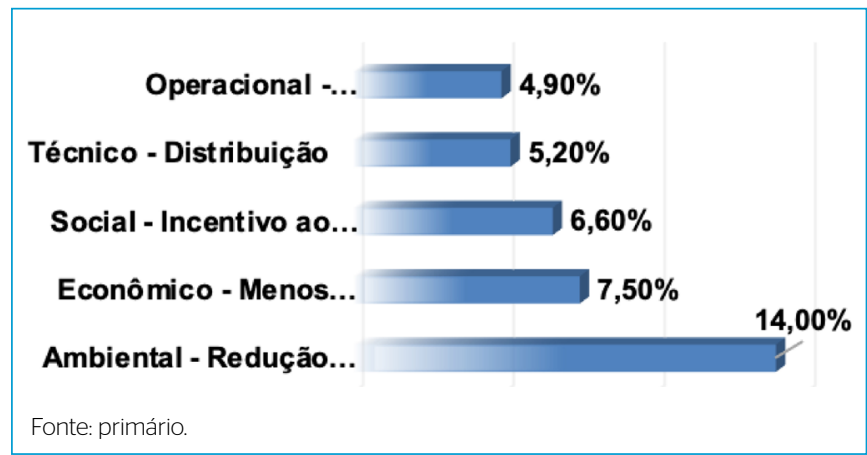

Figura 12 - Resultados dos subcritérios.

De maneira geral, o uso de fontes alternativas não é uma medida de otimização do uso, apenas substituição de fonte. O conceito de otimização está associado a práticas e processos que efetivamente reduzem o consumo da água no empreendimento, o que pode ser verificado pelo consumo específico de água, por exemplo (litros por usuário).

O reúso de água pode ser benéfico em um cenário de escassez de água e para a redução dos custos associados às despesas com água e esgotos da companhia de saneamento local. Já o aproveitamento da água de chuva tem benefício na redução do escoamento superficial e econômico. Segundo Mierzwa (2002), nenhuma dessas opções implica em redução do consumo, pois, como dito anteriormente, a redução do consumo virá do comportamento do usuário; em seguida (e somadas), virão todas as ações e reações da manutenção e operação do shopping.

De forma a concluir, os resultados finais necessitam de uma visão holística, que se inicia na concepção do empreendimento, passando pela execução e pelos demais conceitos de uso consciente. A base de todas as ações está no conhecimento e na aplicação; para isso, os profissionais de projetos devem ter acesso às pesquisas, para que possam pôr em prática seus conhecimentos, aproximando, cada vez mais, o mercado da academia e, com isso, produzindo empreendimentos mais sustentáveis, com ganhos ambientais, sociais e econômicos.

\section{Sugestões para futuras pesquisas}

As respostas não representam a visão nacional, para isso os autores sugerem que trabalhos semelhantes sejam aplicados em outros estados, para se obter um diagnóstico no âmbito nacional sobre as percepções dos atores envolvidos em projetos e gestão de shopping centers. Cada região tem suas peculiaridades em termos de ambiente, procedimentos e cultura; avançar no assunto envolve, necessariamente, atividade, se desprender de uma rotina de visão estática, com ações que nos tiram de uma realidade e nos levam ao próximo estágio.

\section{CONTRIBUIÇÕES DOS AUTORES}

Bastos, C.: Conceituação, Curadoria de Dados, Investigação, Escrita, Metodologia, Software, Visualização, Recursos, Escrita - Primeira Redação, Escrita - Revisão e Edição. Calmon, J. L.: Supervisão, Validação, Administração do Projeto, Análise formal. 


\section{REFERÊNCIAS}

ABEDI, M.; TORABI, S.A.; NOROUZI, G.H. Application of fuzzy AHP method to integrate geophysical data in a prospect scale, a case study: Seridune copper deposit. Bollettino di Geofisica Teorica ed Applicata, Tehran, v. 54, n. 2. p. 145-164, 2013. http://doi.org/10.4430/bgta0085

BELLO, F.L. Água: Cada Vez Mais Escassa. Shopping Centers, São Paulo, v. 1, n. 185, p. 58-60, 2013. Disponível em: https://www.ipt.br/download. php?filename=990-Revista_Shopping_Centers_Outubro_de_2013.pdf. Acesso em: 12 abr. 2018.

BINT, L.; GARNETT, A.; SIGGINS, A.; JAQUES, R. Alternative water sources in New Zealand's commercial buildings. Water Supply, v. 19, n. 2, p. 371-381, 2019. https://doi.org/10.2166/ws.2018.082

BOTTERO, M.; COMINO, E.; RIGGIO, V. Application of the analytic hierarchy process and the analytic network process for the assessment of different wastewater treatment systems. Journal Environmental Modelling \& Software, v. 26, n. 10, p. 1211-1224, 2011. https://doi.org/10.1016/j. envsoft.2011.04.002

BOYLE, C.A. Sustainable buildings. Engineering Sustainability, v. 158, n. 1, p. 41-48, 2005. https://doi.org/10.1680/ensu.2005.158.1.41

CHEN, C.F. Applying the Analytical Hierarchy Process (AHP) Approach to Convention Site Selection. Journal of Travel Research, v. 45, n. 2, p. 167-174, 2006. https://doi.org/10.1177\%2F0047287506291593

COOK, S.; SHARMA, A.K.; GURUNG, T.R. Evaluation of alternative water sources for commercial buildings: A case study in Brisbane, Australia. Resources, Conservation and Recycling, v. 89, p. 86-93, 2014. https://doi. org/10.1016/j.resconrec.2014.05.003

COSTA, J.F.D.S.; RODRIGUES, M.D.M.; FELIPE, A.P.M. Utilização do método de análise hierárquica (AHP) para escolha de interface telefônica. In: ENCONTRO NACIONAL DE ENGENHARIA DE PRODUÇÃO, 28., 2008. Anais [...]. Rio de Janeiro: ABEPRO, 2008. p. 1-14.

DE GOIS, E.H.B.; RIOS, C.A.S.; COSTANZI, R.N. Evaluation of water conservation and reuse: A case study of a shopping mall in southern Brazil. Journal of Cleaner Production, v. 96, p. 263-271, 2015. https://doi.org/10.1016/j. jclepro.2014.08.097

FREIRE, M.T.M. O consumo racional de água no aeroporto internacional de Salvador, Bahia/Brasil. Dissertação (Mestrado em Engenharia Industrial) Universidade Federal da Bahia, Salvador, 2011.

JAGTAP, H.P.; BEWOOR, A.K. Use of Analytic Hierarchy Process Methodology for Criticality Analysis of Thermal Power Plant Equipments. Materials Today Proceedings, Pune, v. 4, n. 2, parte A, p. 1927-1936, 2017. https://doi. org/10.1016/j.matpr.2017.02.038

JOUSTRA, C.; YEH, D. H. Framework for net-zero and net-positive building water cycle management. Building Research and Information, v. 43, n. 1, p. 121-132, 2015. https://doi.org/10.1080/09613218.2015.961002

KIPERSTOK, A. (org.). Prata da casa: construindo produção limpa na Bahia. Salvador: Rede de Tecnologias Limpas (Teclim)/Universidade Federal da Bahia (UFBA), 2008. 446 p.
KURTTILA, M.; PESONEN, M.; KANGAS, J.; KAJANUS, M. Utilizing the analytic hierarchy process (AHP) in SWOT analysis - a hybrid method and its application to a forest-certification case. Forest Policy and Economics, v. 1, n. 1. p. 41-52, 2000. https://doi.org/10.1016/S1389-9341(99)00004-0

MACHARIS, C.; SPRINGAEL, J.; BRUCKER, K.; VERBEKE, A. PROMETHEE and AHP: The design of operational synergies in multicriteria analysis. Strengthening PROMETHEE with ideas of AHP. European Journal of Operational Research, Bruxelas, v. 153, n. 2, p. 307-317, 2004. https://doi. org/10.1016/S0377-2217(03)00153-X

MARINS, F.A.S.; PEREIRA, M.S.; BELDERRAIN, M.C.N.; URBINA, L.M.S. Métodos de tomada de decisão com múltiplos critérios: aplicações na indústria aeroespacial. São Paulo: Blucher Acadêmico, 2010.

MIERZWA, J.C. O Uso Racional e Reúso como Ferramenta para o Gerenciamento de Águas e Efluentes na Indústria Estudo de Caso para a Kodak Brasileira. 399f. Tese (Doutorado) - Universidade de São Paulo, São Paulo, 2002.

NUNES, R.T.S. Conservação da água em edifícios comerciais: potencial de uso racional e reúso em shopping center. Dissertação (Mestrado) Universidade Federal do Rio de Janeiro, Rio de Janeiro, 2006.

RIBEIRO, A.C.; COSTA, H.G. Emprego do método de análise hierárquica (AHP) na distribuição de custos indiretos: uma proposta para a pequena e média empresa. In: ENCONTRO NACIONAL DE ENGENHARIA DE PRODUÇÃO, 19., 1999, Rio de Janeiro, Anais [...]. Rio de Janeiro: UFRJ, 1999.

SOUSA, V.; SILVA, C. M.; MEIRELES, I. Performance of water efficiency measures in commercial buildings. Resources, Conservation and Recycling, v. 143, p. 251-259, 2019. https://doi.org/10.1016/j.resconrec.2019.01.013

SOUSA, V.; SILVA, C.M.; MEIRELES, I.C. Technical-financial evaluation of rainwater harvesting systems in commercial buildings-case ase studies from Sonae Sierra in Portugal and Brazil. Environmental Science and Pollution Research, v. 25, p. 19283-19297, 2018. https://doi.org/10.1007/s11356017-0648-0

TEMIZ, I.; CALIS, G. Selection of Construction Equipment by Using Multicriteria. In: CREATIVE CONSTRUCTION CONFERENCE, 2017. Anais [...]. Primosten: Science Direct, 2017. p. 19-22.

TRENTIM, M. Tomada de Decisão em Projetos - Método AHP. Mundo Project Management, 2012. Disponível em: https://projectdesignmanagement.com. br/blog/tomada-de-decisao-em-projetos/. Acesso em: 19 fev. 2019.

WANG, N.; CHANG, Y.-C.; NUNN, C.R. Lifecycle assessment for sustainable design options of a commercial building in Shanghai. Building and Environment, v. 45, n. 6, p. 1415-1421, 2010. https://doi.org/10.1016/j. buildenv.2009.12.004

WANG, S.; SHENG, Z.; XI, Y.; MA, X.; ZHANG, H.; KANG, M.; REN, F.; DU, Q.; $\mathrm{HU}, \mathrm{K}$.; HAN, Z. The Application of the Analytic Hierarchy Process and a New Correlation Algorithm to Urban Construction and Supervision Using Multi-Source Government Data in Tianjin. ISPRS International Journal of Geo-Information, v. 7, n. 2, p. 50, 2018. https://doi.org/10.3390/ijgi7020050 